\title{
Dynamics of a Delayed Interactive Model Applied to Information Dissemination in Social Networks
}

\author{
Yaming Zhang, ${ }^{1,2}$ Fei Liu $\mathbb{D}^{1,3}$ Yaya H. Koura ${ }^{1}{ }^{1}$, and Yanyuan $^{1,3}{ }^{1}$ \\ ${ }^{1}$ School of Economics and Management, Yanshan University, Qinhuangdao 066004, China \\ ${ }^{2}$ Center for Internet Plus and Industry Development, Yanshan University, Qinhuangdao 066004, China \\ ${ }^{3}$ School of Public Administration, Hengshui University, Hengshui 053000, China \\ Correspondence should be addressed to Fei Liu; liufei937087578@126.com and Yaya H. Koura; 2689395039@qq.com
}

Received 2 November 2020; Revised 7 February 2021; Accepted 25 February 2021; Published 8 March 2021

Academic Editor: Stefano Valvano

Copyright (c) 2021 Yaming Zhang et al. This is an open access article distributed under the Creative Commons Attribution License, which permits unrestricted use, distribution, and reproduction in any medium, provided the original work is properly cited.

Reducing fake news and rumor propagation through social media may be challenging to achieve when dealing with sensible contents and communities with free access to online shared resources. Controlling rumor dissemination and promoting true news are the main techniques used to strangle false information that may result in dramatic effect on human wellbeing in an open or closed environment. In this article, we studied a predator-prey model with constant delay in both predator and prey equations and applied the proposed model to the underlying relationship between the existing rumor propagating through social media and the related authoritative information containing the truth broadcast to reduce the respective rumor negative effect on the targeted community. We showed that the proposed system was very responsive to small perturbations and exhibited complex dynamical behavior around the steady-state equilibrium when interaction occurs and delay is applied, considering the controlled situations. Numerical results suggested applying relatively small delay, which represents the ideal time to publish the related propagating rumor curative content to reduce its diffusion speed and promote the truth.

\section{Introduction}

Preventing disinformation and the proliferation of misleading contents such as fake news circulating through digital media is a big responsibility for online platforms, civil society, and the authority. Each of us plays an important role in ensuring that information is reliable before it is shared. The problem of misinformation on social media has caused widespread alarm as stipulated in recent related literature [1-3].

In this article, we study a two-species modified predatorprey system to model the outcome of the underlying interaction occurring when an authoritative information is broadcast to compete with a dangerous rumor propagating through a given social media platform. This investigation is motivated by observations of rumor recurrence and persistence phenomenon in complex social networks. Particularly, in the period of crisis where sensible contents circulating through a given platform could end up affecting dramatically the respective virtual community mind and social wellbeing, as mentioned above, social interactions are more and more risky and present many forms of threats [4-6].

In population dynamics, delaying the interactive species resources' accessibility, assimilation, or harvesting is one of the most commonly used techniques for controlling overpopulation, massive domination, and overbirth and extinction cycles governing system dynamics. Analyzing the effect of such delaying mechanisms has been extensively studied in related literature $[7,8]$. Models with single delay remain attractive in modeling ecosystems in which the studied species compete for habitat or resources [9-11]. The delaying mechanism was introduced to take into account gestation period, maturation time and latency, or system lags. In some studies, the delay parameter was employed to model the relationship between resources' availability and accessibility or assimilation time, predator harvesting effort, handling time, and so forth. The main characteristics of 
predator-prey models with delay reside in that such models exhibit very complex dynamical behavior and are responsive to small perturbations, allowing more flexibility in control and management for decision makers [12-16]. Such systems also present a special interest in studying hyperbolic and nonhyperbolic critical points or trajectories in system behavior when bifurcation and deterministic chaotic orbits could be found for some set of parameters.

In system engineering, complex networks, and applied sciences, controlling chaos and unpredictable behavior is a common practice, as many authors have investigated. However, deterministic chaotic system could be challenging to control and might be difficult to adapt in the real world as some hidden or uncategorized factors might induce the observed changes and random behavior [17-22]. This is the case for biological, electronic, or chemical reactions' systems. For such systems, unpredictability may signify paradox or complexity. From the mathematical standpoint, it is often assimilated to perturbations due to errors related to parameters' estimation and constitutes a solid evidence and valid reason to test the respective system sensibility to small perturbations.

Nevertheless, in complex networks, economics, and finances, for example, it is common to deal with uncertainty, and there exist many methods for solving such problems, measuring, analyzing, predicting, and avoiding chaos, and so forth. In the case of delay, when it is applied to a given part of the system, it is often feasible using well-known tools and techniques to determine how it affects the main system behavior in short- or long-term prediction. In the case of rumor or disinformation, the persistent rumor will continue propagating even after the release of the related authoritative information. To strengthen the authoritative information competitiveness, publishing it at the right time may result in influencing significantly the persistent fake news by reducing its diffusion in the short- or long-term scale. In the proposed model, we introduced a delay parameter in the interaction terms and a control parameter in the prey species equation to control its speed of diffusion and promote the released true news or information [23-33].

We performed qualitative analysis of the proposed interactive model and found that, at certain conditions, a stable positive equilibrium state could be determined in case of coexistence. The system exhibited rich dynamical behavior as stable and periodic trajectories could be found in both prey and predator dynamics. Results of numerical simulations suggested applying relatively small delays and promoting the authoritative information to enhance its competitiveness and reduce the related rumor negative effect on the targeted community.

\section{Preliminaries}

Let $X$ and $Y$ be, respectively, the prey and predator population size at time $t$. We assume the studied system has unlimited resources for analysis purposes, and as in classical Lotka-Volterra-based models, all resources are available and accessible, intensifying inner and intercompetition. In this approach, $Y$ will relatively increase its population size when there is abundance of $X$ depending on control parameters. Prey high population size will allow $Y$ to grow faster depending on its ability or strength, assimilated here to the broadcast message content attractiveness, promotion by the authority, etc. In nature and complex networks, prey strong presence may indicate its ability to escape predation or defend itself. This may also indicate its adaptation to changes, say people are more likely to search and read, respectively, the trending rumor or the authoritative information driven by curiosity, for example. The trending rumors may be presented such that they appear more attractive and convincing for people with average cognitive ability or discernment. To take into consideration these observations, we use a delay parameter in the interaction terms of the proposed system. Delaying the interaction terms formalizes the initial historic function or attractiveness of the respective propagating rumor before the release of the related authoritative information. The delay could also indicate that the newly published true information needs time to convince and to benefit in a number of views from the positive feedback. Meanwhile, the propagating rumor will continue increasing views for a certain time after the release of the related authoritative information.

A model system is given by

$$
\begin{aligned}
& \left\{\begin{array}{l}
\frac{\mathrm{d} X}{\mathrm{~d} t}=r_{1} X(a-X)-b X(t-\tau) Y-c X^{3}, \\
\frac{\mathrm{d} Y}{\mathrm{~d} t}=r_{2} X(t-\tau) Y-e Y^{2},
\end{array}\right. \\
& t \in\left[t_{0}-\tau, t_{0}\right], \quad \tau>0 ; \\
& X(t)=\Phi(t)>0 ; \quad X(0)>0, Y(0)>0 ; \\
& r_{1}, r_{2}, a, b, c, e>0,
\end{aligned}
$$

where $r_{1}$ represents $X$ growth factor. This factor measures the rate of increase in the number of views directly related to users' activities when they repost or transmit the respective rumor. $a$ is a control parameter related to the number of views of the trending rumor in respect to the total number of views at time $t$ on the studied platform. $b$ and $r_{2}$ are, respectively, $Y$ interaction factor and a rate of increase due to the positive feedback capturing the per capita density decrease or increase per unit time. In this approach, we consider that people who enter in contact with the authoritative information know about the current related trending rumor. $c$ and $e$ are, respectively, $X$ and $Y$ decay factors when they shrink size for numerous possible reasons, starting from human forgetting, decrease in interest, appearance of new rumors or captivating news, and so forth.

$\Phi(t)$ represents the initial data or historic function formalizing the trending rumor propagation record before the release of the related authoritative information. This parameter can be adjusted to promote the authoritative information over the current persistent rumor for security and social wellbeing purposes. $\tau$ is assumed to be 
proportional to the authoritative information release date and $X$ state before the release of $Y$.

For $\tau=0$, system (1) becomes

$$
\left\{\begin{array}{l}
\frac{\mathrm{d} X}{\mathrm{~d} t}=r_{1} X(a-X)-b X Y-c X^{3}=f_{1}(X, Y), \\
\frac{\mathrm{d} Y}{\mathrm{~d} t}=r_{2} X Y-e Y^{2}=f_{2}(X, Y) .
\end{array}\right.
$$

For $f_{1}(X, Y)>0$ and $f_{2}(X, Y)>0$, we have

$$
\begin{aligned}
& r_{1}(a-X)-b Y-c X^{2}>0, \\
& c X^{2}+r_{1} X<a r_{1}-b Y, \\
& a r_{1}-b Y>0, \\
& Y<\frac{a r_{1}}{b}, \longrightarrow b Y=\varepsilon, \\
& c X^{2}+r_{1} X+\varepsilon<0, \\
& \frac{-r_{1}-\sqrt{r_{1}^{2}-4 c\left(\varepsilon-a r_{1}\right)}}{2 c} \leq X(t) \\
& \leq \frac{-r_{1}+\sqrt{r_{1}^{2}-4 c\left(\varepsilon-a r_{1}\right)}}{2 c}, \\
& 0<Y(t)<\frac{r_{1}}{b} .
\end{aligned}
$$

For $f_{1}(X, Y)<0$ and $f_{2}(X, Y)>0$, which is the ideal scenario where $Y$ thrives and strangles $X$, we have

$$
\begin{aligned}
& 0<Y(t)<\frac{r_{2} X(t)}{e}, \\
& 0<Y(t)<\frac{r_{1}\left(r_{1}+4 a c\right)}{4 b c} .
\end{aligned}
$$

This implies that the solutions of system (2) are bounded when both $X$ and $Y$ are attractive to the readership.

If we consider that $f_{1}, f_{2} \longrightarrow C^{2}$ on the phase plane of the system, then

$$
\begin{aligned}
\frac{\partial f_{1}}{\partial X} & =a r_{1}-2 a r_{1} X-b Y-3 c X^{2} \longrightarrow\left|\frac{\partial f_{1}}{\partial X_{1}}\right| \\
& =\left|a r_{1}-\left(2 a r_{1} X+b Y+3 c X^{2}\right)\right|<a r_{1} \\
\frac{\partial f_{2}}{\partial Y} & =r_{2} X-2 e Y \longrightarrow\left|\frac{\partial f_{2}}{\partial Y}\right|=\left|r_{2} X-2 e Y\right| \leq r_{2}|X| \\
\frac{\partial^{2} f_{1}}{\partial X^{2}} & =-2 a r_{1}-6 c X \\
\frac{\partial^{2} f_{2}}{\partial Y^{2}} & =-2 e
\end{aligned}
$$

Based on the Lipschitz condition, all positive solutions of the system are defined, bounded, and unique $\forall t>0$.
Furthermore, computing the divergence and the Laplacian, we get

$$
\begin{aligned}
\nabla\left(f_{1}, f_{2}\right)= & \frac{\partial f_{1}}{\partial X}+\frac{\partial f_{2}}{\partial Y}=a r_{1}+r_{2} X \\
& -\left[3 c X^{2}+2 a r_{1} X+(b+2 e) Y\right] \neq 0, \\
\nabla^{2}\left(f_{1}, f_{2}\right)= & \frac{\partial^{2} f_{1}}{\partial X^{2}}+\frac{\partial^{2} f_{2}}{\partial Y^{2}}=-2\left(3 c X+a r_{1}+e\right)<0 .
\end{aligned}
$$

Clearly, $\nabla\left(f_{1}, f_{2}\right)$ could be negative or positive depending on the parameters' value; therefore, system (2) does admit periodic solutions on the phase plane according to the Poincaré-Bendixson criterion for some sets of parameter values.

Solving (2), we get

$$
\begin{aligned}
Y^{*}= & \frac{r_{2} X^{*}}{e},-c X^{2}-X\left(r_{1}+\frac{b r_{2}}{e}\right)+a r_{1}=0, \\
X^{*}= & -\frac{1}{2 c}\left[r_{1}+\frac{b r_{2}}{e}-\sqrt{\left.\left(r_{1}+\frac{b r_{2}}{e}\right)^{2}+4 a c r_{1}\right]}>0,\right. \\
\left(X^{*}, Y^{*}\right)= & -\frac{1}{2 c}\left[r_{1}+\frac{b r_{2}}{e}-\sqrt{\left(r_{1}+\frac{b r_{2}}{e}\right)^{2}+4 a c r_{1}}\right], \\
& \left.-\frac{r_{2}}{2 c e}\left[r_{1}+\frac{b r_{2}}{e}-\sqrt{\left(r_{1}+\frac{b r_{2}}{e}\right)^{2}+4 a c r_{1}}\right]\right) .
\end{aligned}
$$

The system admits a unique positive equilibrium point $\left(X^{*}, Y^{*}\right)$ in case of the coexistence of $X$ and $Y$.

3. System Behavior for $\tau==0$

(1) The Jacobian matrix is given by

$$
J=\left(\begin{array}{cc}
a r_{1}-X\left(2 r_{1}+3 c X\right)-b Y & -b X \\
r_{2} Y & r_{2} X-2 e Y
\end{array}\right) .
$$

Evaluated at the origin, we obtain

$$
J(0,0)=\left(\begin{array}{cc}
a r_{1} & 0 \\
0 & 0
\end{array}\right)
$$

The origin is a saddle unstable node as the determinant is zero and the trace is always positive.

At $\left(X^{*}, Y^{*}\right)$, we have

$$
\begin{aligned}
T= & a r_{1}+r_{2} X^{*}-\left[X^{*}\left(2 r_{1}+3 c X^{*}\right)+Y^{*}(b+2 e)\right], \\
D= & {\left[a r_{1}-X^{*}\left(2 r_{1}+3 c X^{*}\right)-b Y^{*}\right] } \\
& \left(r_{2} X^{*}-2 e Y^{*}\right)+b r_{2} X^{*} Y^{*} .
\end{aligned}
$$


$\left(X^{*}, Y^{*}\right)$ is locally asymptotically stable attracting all nearby trajectories if we can ensure $T<0$ and $D>0$ according to the Routh-Hurwitz stability criterion. This could be satisfied if (11) holds:

$$
\begin{aligned}
& \left\{\begin{array}{l}
a r_{1}<X^{*}\left(2 r_{1}+3 c X^{*}\right)+b Y^{*}, \\
r_{2} X^{*}<2 e Y^{*},
\end{array}\right. \\
& \frac{Y^{*}}{X^{*}}>\frac{r_{2}}{e} .
\end{aligned}
$$

(2) In case the polynomial characteristic equation admits complex conjugate roots of the form $\lambda=\alpha+i \beta$, then we will have

$$
\begin{aligned}
P(\lambda)= & \alpha^{2}-\beta^{2}+2 i \alpha \beta-\alpha\left(a r_{1}+r_{2} X^{*}\right)+\alpha\left(2 r_{1} X^{*}+b Y^{*}+3 c X^{*}+2 e Y^{*}\right) \\
& -i \beta\left(a r_{1}+r_{2} X^{*}\right)+i \beta\left(2 r_{1} X^{*}+b Y^{*}+3 c X^{*}+2 e Y^{*}\right) \\
& +\left(a r_{1}-b Y^{*}-3 c X^{* 2}-2 r_{1} X^{*}\right)\left(r_{2} X^{*}-2 e Y^{*}\right)+b r_{2} X^{*} Y^{*}=0, \\
\operatorname{Re}(\lambda)= & \alpha^{2}-\beta^{2}+2 i \alpha \beta-\alpha\left(a r_{1}+r_{2} X^{*}\right)+\alpha\left(2 r_{1} X^{*}+b Y^{*}+3 c X^{*}+2 e Y^{*}\right) \\
& +\left(a r_{1}-b Y^{*}-3 c X^{* 2}-2 r_{1} X^{*}\right)\left(r_{2} X^{*}-2 e Y^{*}\right)+b r_{2} X^{*} Y^{*}=0, \\
\operatorname{Im}(\lambda)= & 2 \alpha-\left(a r_{1}+r_{2} X^{*}\right)+\left(2 r_{1} X^{*}+b Y^{*}+3 c X^{*}+2 e Y^{*}\right)=0 .
\end{aligned}
$$

For $\alpha=0$,

$$
\begin{aligned}
\operatorname{Re}(\lambda) & =-\beta^{2}+\left(a r_{1}-b Y^{*}-3 c X^{* 2}-2 r_{1} X^{*}\right)\left(r_{2} X^{*}-2 e Y^{*}\right)+b r_{2} X^{*} Y^{*}=0 \\
\operatorname{Im}(\lambda) & =-\left(a r_{1}+r_{2} X^{*}\right)+\left(2 r_{1} X^{*}+b Y^{*}+3 c X^{*}+2 e Y^{*}\right)=0 \\
\beta & = \pm \sqrt{q} \\
q & =\left(a r_{1}-b Y^{*}-3 c X^{* 2}-2 r_{1} X^{*}\right)\left(r_{2} X^{*}-2 e Y^{*}\right)+b r_{2} X^{*} Y^{*}>0
\end{aligned}
$$

If (13) could be satisfied, then traveling curves, when approaching the steady-state equilibrium, should exhibit periodic motion with relative amplitude depending on $\beta$.

For $\alpha \neq 0$,

$$
\begin{aligned}
R= & \alpha^{2}-\alpha\left(r_{1} a+r_{2} X^{*}\right)+\alpha\left(2 r_{1} X^{*}+b Y^{*}+3 c X^{*}+2 e Y^{*}\right) \\
& +\left(a r_{1}-2 r_{1} X^{*}-b Y^{*}-3 c X^{* 2}\right)\left(2 e Y^{*}-r_{2} X^{*}\right) \\
& +b r_{2} X^{*} Y^{*}>0, \\
\beta= & \pm \sqrt{R} .
\end{aligned}
$$

If (14) could be satisfied, then $\left(X^{*}, Y^{*}\right)$ would behave as a spiral unstable node $(\alpha>0)$. In this case, traveling trajectories would have outward motion with growing amplitude depending on $\beta$.

(3) Global behavior:

Suppose $V(X, Y)$ is a Lyapunov function for the system, $V(X, Y)$ is given by

$$
V(X, Y)=\frac{X^{4}}{X+1}+\frac{Y^{2}}{Y+1}+h X^{2} Y
$$

Then,

$$
\begin{aligned}
V(0,0)= & 0 \\
V(X, Y)= & \frac{X^{4}}{X+1}+\frac{Y^{2}}{Y+1}+h X^{2} Y>0 \\
& \longrightarrow X>0, Y>0,0=h<1 .
\end{aligned}
$$

Then, differentiating with respect to time yields

$$
\begin{aligned}
V^{\prime}(X, Y)= & X^{2}\left(\frac{3 X^{3} 4 X^{3}}{(X+1)^{2}}+2 h Y\right)\left(a r_{1}-r_{1} X-b Y-c X^{2}\right) \\
& +Y\left(\frac{Y(Y+2)}{(Y+1)^{2}}+h X^{2}\right)\left(r_{2} X-e Y\right) .
\end{aligned}
$$

$V^{\prime}(X, Y)$ is negative definite if $a r_{1}<r_{1} X+b Y+c X^{2}$ and $r_{2} X<e Y$, which implies 


$$
\left\{\begin{array}{l}
\left(X+\frac{1}{2 c}\left[r_{1}-\sqrt{r_{1}^{2}+4 a c r_{1}}\right]\right)\left(X+\frac{1}{2 c}\left[r_{1}+\sqrt{r_{1}^{2}+4 a c r_{1}}\right]\right)>b Y \\
r_{2} X<e Y
\end{array}\right.
$$

Furthermore, $V$ is radially unbounded as

$$
\lim _{t \rightarrow \infty, \forall h} V(X, Y) \longrightarrow \infty .
$$

$$
\begin{aligned}
& V(X, Y)-V\left(X^{*}, Y^{*}\right)=\left(\frac{X^{* 3}\left(3 X^{*}+4\right)}{\left(X^{*}+1\right)^{2}}\right)\left(X-X^{*}\right)+\left(\frac{Y^{*}\left(Y^{*}+2\right)}{\left(Y^{*}+1\right)^{2}}\right)\left(Y-Y^{*}\right) \\
& +\left(\frac{X^{* 2}\left(3 X^{* 2}+8 X^{*}+6\right)}{\left(X^{*}+1\right)^{3}}\right)\left(X-X^{*}\right)^{2}+\frac{\left(Y-Y^{*}\right)^{2}}{\left(Y^{*}+1\right)^{3}} \\
& =X^{* 2}\left(X-X^{*}\right)\left[\frac{X^{*}\left(3 X^{*}+4\right)\left(X^{*}+1\right)+\left(3 X^{* 2}+8 X^{*}+6\right)\left(X-X^{*}\right)}{\left(X^{*}+1\right)^{3}}\right] \\
& +\left(Y-Y^{*}\right)\left[\frac{Y^{* 3}+3 Y^{* 2}+Y-Y^{*}}{\left(Y^{*}+1\right)^{3}}\right] \\
& =X^{* 2}\left(X-X^{*}\right)\left[\frac{X^{* 2}\left(3 X^{*}-1\right)+2 X^{*}(4 X-1)+6 X}{\left(X^{*}+1\right)^{3}}\right] \\
& +\left(Y-Y^{*}\right)\left[\frac{Y^{* 3}+3 Y^{* 2}+Y-Y^{*}}{\left(Y^{*}+1\right)^{3}}\right]
\end{aligned}
$$

If we chose $h \approx 0$, then

$$
V(X, Y)-V\left(X^{*}, Y^{*}\right)<0 \rightarrow X^{*}>\frac{1}{3}, Y^{*}>\frac{1}{3} .
$$

$V(X, Y)-V\left(X^{*}, Y^{*}\right)$ has a definite negative sign, implying the global attractiveness of $\left(X^{*}, Y^{*}\right)$. If we can maintain $X^{*}<1, Y^{*}<1 / 3$, then $\left(X^{*}, Y^{*}\right)$ would be globally asymptotically stable according to the Lyapunov theorem.

\section{Model Stability Analysis for $\tau>0$}

System (1) is considered as a single constant delayed system. The corresponding transcendental polynomial characteristic could be determined as follows: 


$$
\left|\begin{array}{cc}
M-b m_{0} Y^{*} e^{-\lambda \tau}-\lambda & -b m_{1} e^{-\lambda \tau} \\
+r_{2} m_{2} Y^{*} e^{-\lambda \tau} & r_{2} m_{3} Y^{*} e^{-\lambda \tau}-2 e Y^{*}-\lambda
\end{array}\right|=0, \quad m_{0}, m_{1}, m_{2}, m_{3}>0, M=a r_{1}-2 r_{1} X^{*}-3 c X^{* 2} \text {. }
$$

It follows that

$$
\begin{aligned}
\lambda^{2} & +\left(2 e Y^{*}-M\right) \lambda+\left(r_{2} m_{3} M+2 e b m_{0} Y^{* 2}+b m_{0} Y^{*} \lambda\right) e^{-\lambda \tau}-2 e M Y^{*} \\
& +b r_{2} Y^{*}\left(m_{1} m_{2}+m_{0} m_{3}\right) e^{-2 \lambda \tau}=0 .
\end{aligned}
$$

Suppose (23) admits complex conjugate purely imaginary roots of the form $\lambda= \pm i \beta$. Then,

$$
\begin{aligned}
& -\beta^{2}+i \beta\left(2 e Y^{*}-M\right)+\left(r_{2} m_{3} M+2 e b m_{0} Y^{* 2}+b m_{0} Y^{*} \lambda\right) e^{-i \beta \tau}-2 e M Y^{*} \\
& +b r_{2} Y^{*}\left(m_{1} m_{2}+m_{0} m_{3}\right) e^{-2 i \beta \tau}=0, \\
& \operatorname{Re}(\lambda)=-\beta^{2}+\left(r_{2} m_{3} M+2 e b m_{0} Y^{* 2}\right) \cos \beta \tau-b m_{0} Y^{*} \beta \sin \beta \tau-2 e M Y^{*} \\
& +b r_{2} Y^{*}\left(m_{1} m_{2}+m_{0} m_{3}\right) \cos 2 \beta \tau=0, \\
& \operatorname{Im}(\lambda)=2 e Y^{*}-M-\left(r_{2} m_{3} M+2 e b m_{0} Y^{* 2}\right) \sin \beta \tau+b m_{0} Y^{*} \beta \cos \beta \tau \\
& -b r_{2} Y^{*}\left(m_{1} m_{2}+m_{0} m_{3}\right) \sin 2 \beta \tau=0 .
\end{aligned}
$$

It follows at the origin

$$
\begin{aligned}
& \operatorname{Re}(\lambda)=-\beta^{2}+r_{2} m_{3} M \cos \beta \tau=0, \\
& \operatorname{Im}(\lambda)=-M-r_{2} m_{3} M \sin \beta \tau=0, \\
& \sin \beta \tau=-\left(r_{2} m_{3}\right)^{-1}<0, \\
& \cos \beta \tau=\frac{\beta^{2}}{r_{2} m_{3} M}>0,
\end{aligned}
$$

$$
\frac{3 \pi}{2 \beta}<\tau^{*}<\frac{2 \pi}{\beta}+2 k \pi, \quad k=1,2,3, \ldots
$$

Evaluated at the coexistence equilibrium or at the vicinity of $\left(X^{*}, Y^{*}\right)$, set

$$
\begin{aligned}
& u_{0}=-\beta^{2}-2 e M Y^{*}, \\
& u_{1}=r_{2} m_{3} M+2 e b m_{0} Y^{* 2}, \\
& u_{2}=-b m_{0} \beta Y^{*} \\
& u_{3}=r_{2} b Y^{*}\left(m_{1} m_{2}-m_{0} m_{3}\right), \\
& u_{4}=2 e Y^{*}-M, \\
& u_{5}=r_{2} m_{3} M+2 e b m_{0} Y^{* 2}
\end{aligned}
$$

Rewriting (24), we obtain $\left\{\begin{array}{l}u_{0}+u_{1} \cos \beta \tau+u_{2} \sin \beta \tau+u_{3} \cos 2 \beta \tau=0 \\ u_{4}-\sin \beta \tau-u_{2} \cos \beta \tau-u_{3} \sin 2 \beta \tau=0\end{array}\right.$

After transformation,

$\left\{\begin{array}{l}u_{0}+u_{1} \cos \beta \tau+u_{2} \sin \beta \tau+2 u_{3} \cos ^{2} \beta \tau-2 u_{3}=0 \\ u_{4}-\sin \beta \tau-u_{2} \cos \beta \tau-2 u_{3} \cos \beta \tau \sin \beta \tau=0\end{array}\right.$

Solving (29) yields

$$
\begin{aligned}
\sin \beta \tau= & \frac{u_{4}-u_{2} \cos \beta \tau}{1+2 u_{3} \cos \beta \tau}, \\
& u_{0}+2 u_{0} u_{3} \cos \beta \tau+u_{1} \cos \beta \tau+2 u_{1} u_{3} \cos ^{2} \beta \tau \\
& +u_{2} u_{4}-u_{2}^{2} \cos \beta \tau \\
& +2 u_{3} \cos ^{2} \beta \tau+4 u_{3}^{2} \cos ^{3} \beta \tau-4 u_{3}^{2} \cos \beta \tau-2 u_{3}=0 .
\end{aligned}
$$

If we set $x=\cos \beta \tau$, then

$$
\begin{aligned}
& 4 u_{3}^{2} x^{3}+2 u_{3}\left(1+2 u_{1}\right) x^{2}+\left(2 u_{0} u_{3}+u_{1}-u_{2}^{2}-4 u_{3}^{2}\right) x-u_{0} \\
& +u_{2} u_{4}=0
\end{aligned}
$$

Changing the variables, 


$$
\begin{aligned}
x= & \Omega-\frac{\left(1+2 u_{1}\right)}{6 u_{3}}, \\
\Omega= & x+\frac{\left(1+2 u_{1}\right)}{6 u_{3}}, \\
p= & \frac{1}{12 u_{3}}\left(1-u_{1}+2 u_{0} u_{3}-u_{2}^{2}-4 u_{3}^{2}\right), \\
q= & \frac{1}{108 u_{3}^{3}}\left[\left(1+2 u_{1}\right)^{3}-2\left(1+2 u_{1}\right)\right. \\
& \left.\left(2 u_{0} u_{3}+u_{1}-u_{2}^{2}-4 u_{3}^{2}\right)-27 u_{3}\left(u_{2} u_{4}-u_{0}\right)^{2}\right] .
\end{aligned}
$$

This yields to the depressed cubic equation as follows:

$$
\Omega^{3}+p \Omega+q=0 .
$$

The solutions of (31) could be computed as follows:

$$
\begin{aligned}
x_{j} & =\Omega_{j}-\frac{\left(1+2 u_{1}\right)}{6 u_{3}}, \quad j=1,2,3 . \\
\cos \beta \tau & =x_{j} \\
\sin \beta \tau & =\frac{u_{4}}{1+2 u_{3} x_{j}}-\frac{u_{2} x_{j}}{1+2 u_{3} x_{j}} .
\end{aligned}
$$

For $\cos \beta \tau=x_{j}>0, u_{4}>0$, and $u_{4}>u_{2} x_{j}$, we have

$$
\tau^{*}<\frac{\pi}{2 \beta}+2 k \pi, \quad k=1,2,3, \ldots
$$

If $u_{4}>0$ and $u_{4}<u_{2} x_{j}$, we have

$$
\frac{3 \pi}{2 \beta}<\tau^{*}<\frac{2 \pi}{\beta}+2 k \pi, \quad k=1,2,3, \ldots
$$

For $\cos \beta \tau=x_{j}<0, u_{4}>0$, and $u_{4}>u_{2} x_{j}$, we have

$$
\frac{3 \pi}{2 \beta}<\tau^{*}<\frac{2 \pi}{\beta}+2 k \pi, \quad k=1,2,3, \ldots
$$

For $\cos \beta \tau=x_{j}=0, u_{4}>0$, and $u_{4}>u_{2} x_{j}$, we have

$$
\tau^{*}=\frac{\pi}{\beta}+2 k \pi, \quad k=1,2,3, \ldots
$$

For $\cos \beta \tau=x_{j}=0, u_{4}<0$, and $u_{4}<u_{2} x_{j}$, we have

$$
\tau^{*}=-\frac{\pi}{\beta}+2 k \pi, \quad k=1,2,3, \ldots
$$

For $t \in\left[t_{0}-\tau, t_{0}\right], \tau>0$, trajectories will orbit around the steady-state equilibrium $\left(X^{*}, Y^{*}\right)$ with variable amplitude depending on the parameters' value, initial conditions, or the historic function. The historic function has to be given or computed from the data at hand using existing solvers or tools. In this particular case, it represents $X$ number of views at $t_{0}$ proportional to $Y$ release time.

\section{Numerical Results}

We carried out numerical simulations to test how responsive the proposed model is to small perturbations and validate the initial theoretical hypotheses. The main purpose is to determine how the system behaves when we vary initial conditions, interaction coefficients, and the delay directly related to the release time and promotion of the authoritative information published to strangle the respective persistent rumor. With data extracted from popular social media, Weibo, WeChat, and Facebook, for example, one can determine, after rescaling, respectively, $r_{1}$ and $r_{2}$ taking into account the total number of views, the rate of increase per unit time, which can be per day, per hour, or per week depending on the studied problem, larger value indicating exponential growth, etc. The decay factors should be proportional to per capita decrease in the number of views per unit time. $a$ being a control parameter, its value could be adjusted according to the expected results. Parameters $c, e$, and $\tau$ would be relatively challenging to determine as they could be composite for many systems and attributing the observed lags or latency for $\tau$ could be difficult, depending on many observable or hidden factors.

Figures 1 and 2 display the system behavior (time series) and phase space when the steady-state equilibrium is locally and asymptotically stable. Both $X$ and $Y$ reach the steady state and remain stable over time. This is the ideal classical situation for many simple nonlinear systems. When both the trending rumor and the authoritative information present equal interest for the public, the outcome of interaction is predictable as far as the delay is reasonably smaller, meaning $Y$ was published at the right time; here, $\tau=0.998$ and $\tau=6.6$; and no random event occurs.

The amplitude of oscillations is highly submitted to the delay value for both species as portrayed in Figure 3. Particularly, with $\tau=3.42$, the authoritative information has a significant effect on the trending rumor, making the truth available for the targeted community. However, the rumor does not die out as it contains a strong argument still attractive for people who hear about it, and they choose to check for themselves, at least by curiosity. It is very hard to eradicate highly appealing content, especially when it nourishes people's dream or hope. Solutions approaching the steady state, in this case, are periodic with variable amplitude depending on initial conditions and control parameters.

In Figure 4, a solution for the system with $\tau=0$ is illustrated. In this scenario (in the same precedent condition portrayed in Figure 3), it is shown that the system does not admit periodic orbits. When more and more people read the trending harmful rumor, the unique positive equilibrium point attracts all traveling trajectories at relative speed depending on control parameters and initial conditions.

For the authoritative information containing a curative content to reach out a larger number of people in the targeted community, one needs to release $Y$ at the ideal time, find a way to stimulate people interest, and, at the same time, provide simple, attractive, and yet informative content. 


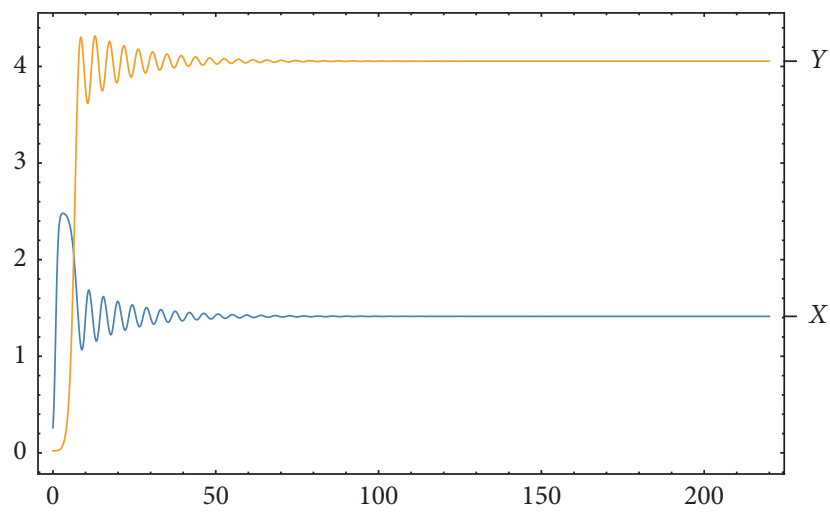

(a)

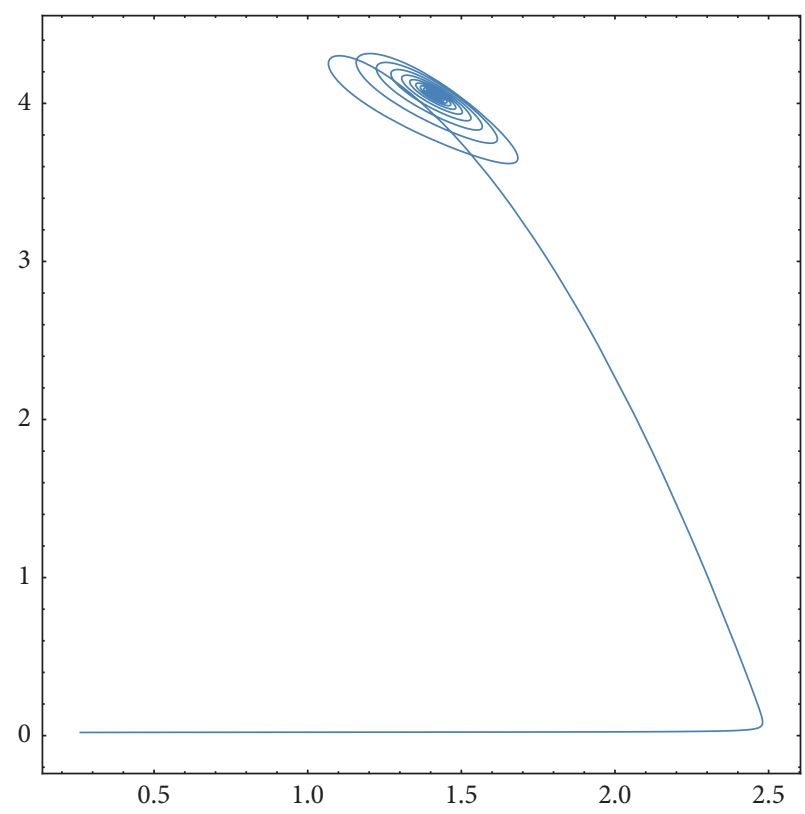

(b)

FIGURE 1: System dynamics showing asymptotically stable behavior at the vicinity of the steady-state equilibrium for $X(0)=0.26$; $Y(0)=0.02$; $r_{1}=0.98 ; a=2.598 ; b=0.279 ; c=0.015 ; r_{2}=0.439 ; e=0.153 ; \tau=0.998$.

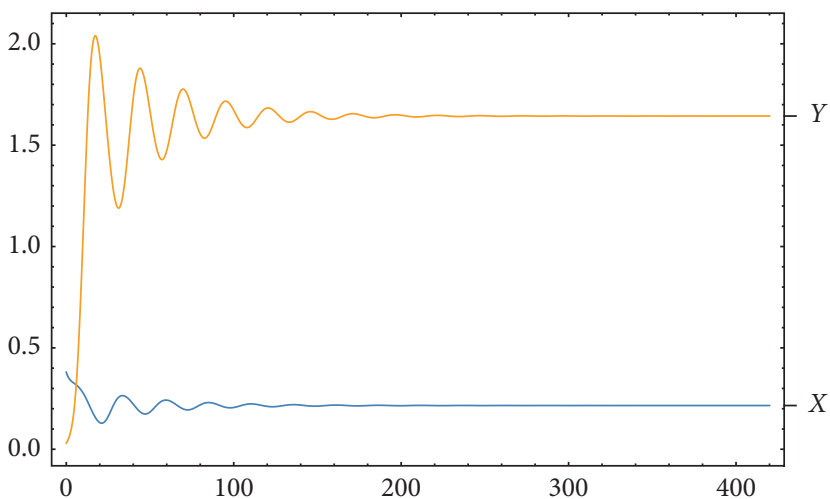

(a)

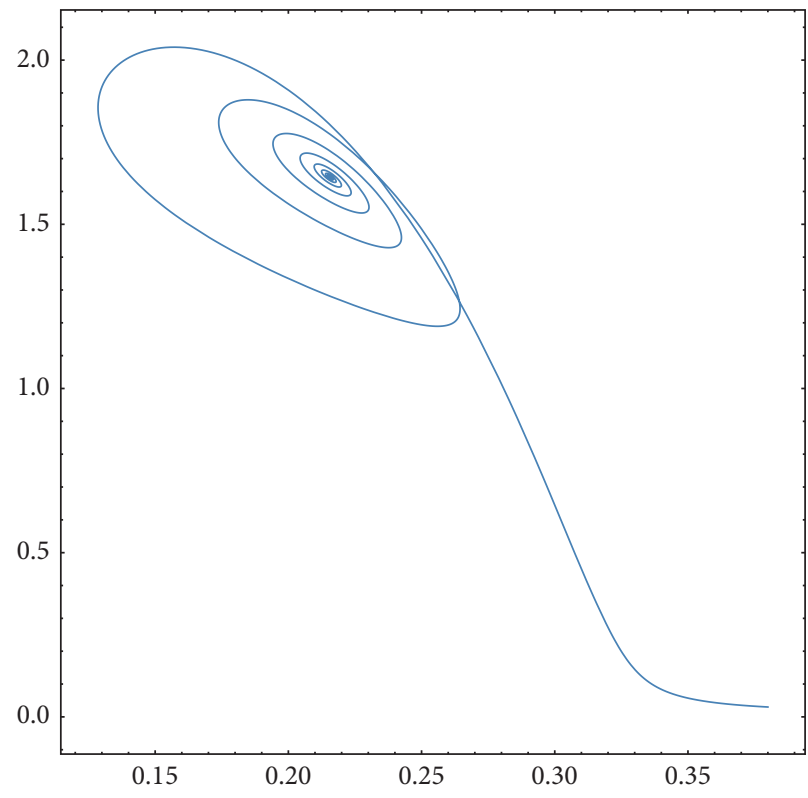

(b)

FIGURE 2: System positive solutions converge to the unique positive equilibrium for $X(0)=0.38 ; Y(0)=0.03 ; r_{1}=1.31 ; a=0.3298 ; b=0.09$; $c=0.035 ; r_{2}=1.09 ; e=0.143 ; \tau=6.6$.

Figure 5 displays a solution curve where the system admits periodic solutions and a stable limit cycle lying at the vicinity of the steady-state equilibrium. This is a desired situation from the control standpoint, with the ultimate goal of strengthening $Y$ competitiveness and attractiveness and keeping reasonably lower $X$ speed of propagation.

However, the delay value has to be chosen such that the stability condition is satisfied in order to allow peaceful coexistence as in Figure 6, where the harmful rumor does not grow exponentially, invading the entire community mind.

To maintain a stable coexistence and promote the authoritative information, there must be enough people reading and transmitting the current related trending rumor. This is consistent with the real situation where readers or the targeted community's interests have to be stimulated. The rumor content, concept, or idea could be used as stimuli 


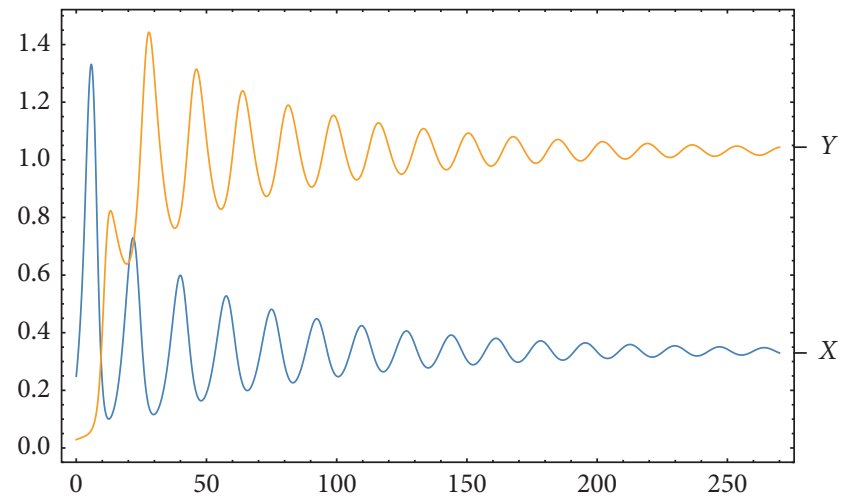

(a)

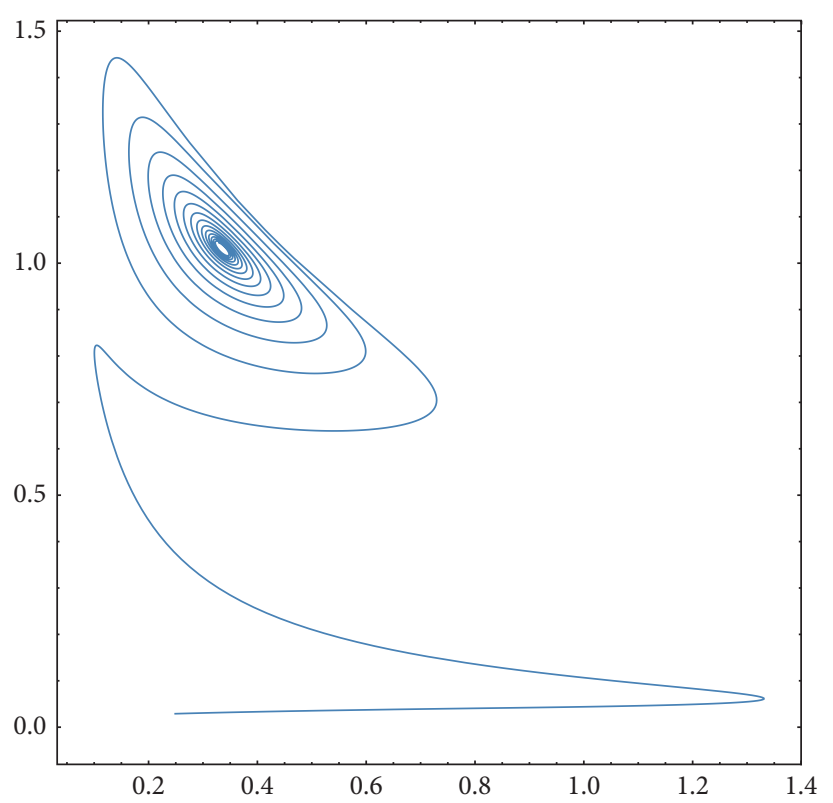

(b)

Figure 3: The system admits periodic solutions for $X(0)=0.249 ; Y(0)=0.029 ; r_{1}=0.88 ; a=0.68 ; b=0.29 ; c=0.035 ; r_{2}=0.439 ; e=0.143$; $\tau=3.42$.

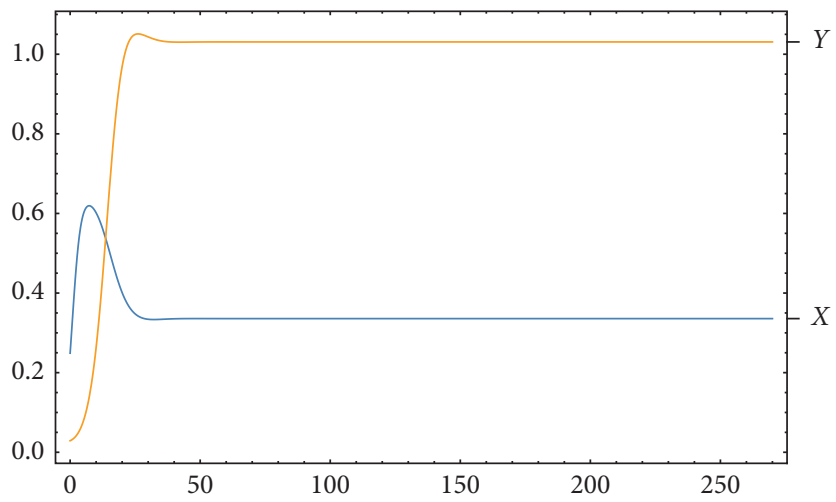

FiguRE 4: The system is locally stable around $\left(X^{*}, Y^{*}\right)$ when no delay is applied for $X(0)=0.249 ; Y(0)=0.029 ; r_{1}=0.88 ; a=0.68 ; b=0.29$; $c=0.035 ; r_{2}=0.439 ; e=0.143 ; \tau=0$.

to attract more people towards discovering the true information.

\section{Discussion}

The results presented in this article could be utilized to support decisions related to the release time of the authoritative information and the effort of the authority to rebuild people trust and confidence in public media by tackling individuals who have taken advantage of social media to create or disseminate fake news. Promoting the authoritative information over the current persistent rumor is challenging, especially when dealing with highly appealing concepts or ideas nourishing people false hope. Delaying the competition terms in the prey equation has the effect of giving more time to the predator to assimilate the harvested resources, here number of views. This will end up strengthening the authoritative information attractiveness. This can be assimilated in real social networks by web marketing tools and techniques to stimulate the targeted people in discovering the real information.

This article presents a new angle of analysis and conceptualization for the recurrent problem of disinformation and benefits of digital media for building people trust and social wellbeing strategies. This investigation also provides, from system thinking and system control standpoint, an insight into numerous factors that may affect the decisionmaking process in this regard. Rumor persistence and the eventual failure of the authoritative information to dissipate related rumors can be explained by many factors. The proposed model could be applied to both autonomous or delayed competitive systems. Lastly, the proposed model system could be extended to higher-dimensional systems, where competition for resources governs the underlying 


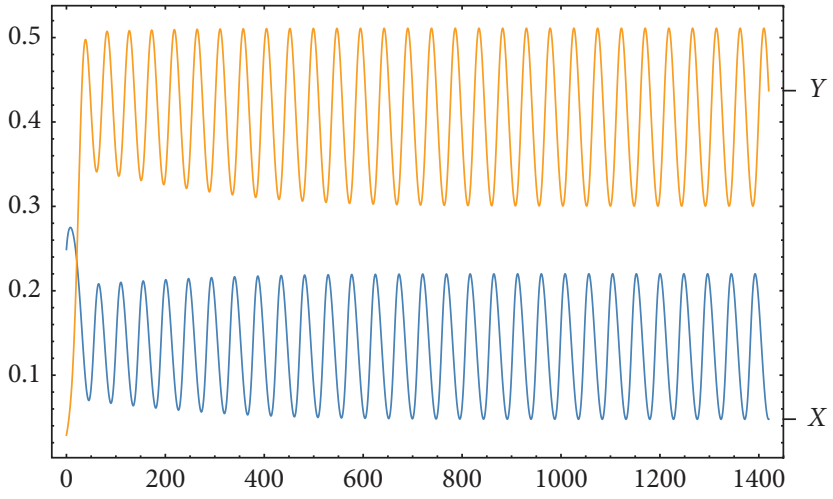

(a)

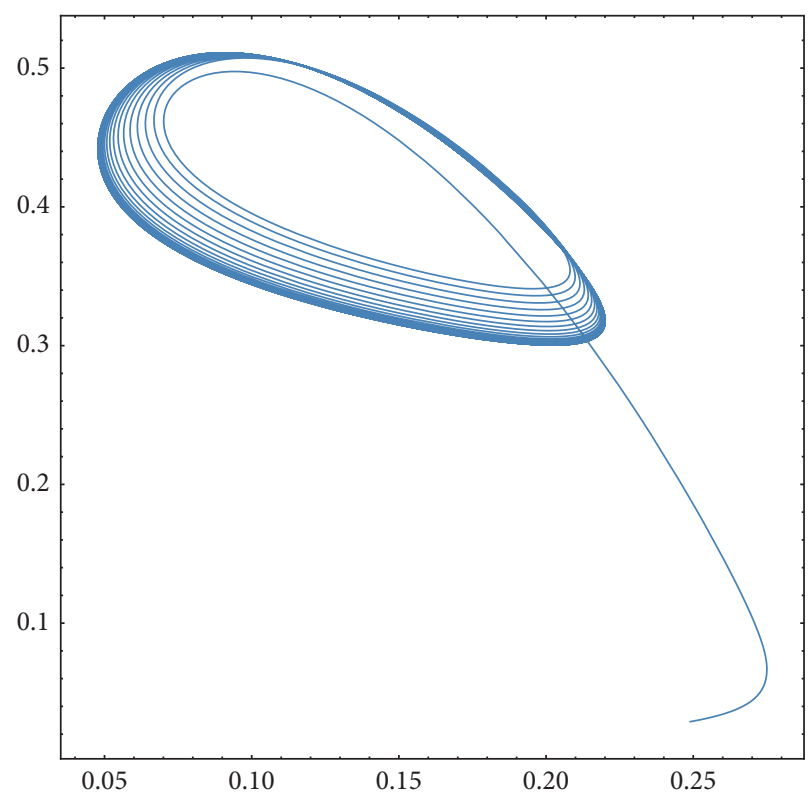

(b)

Figure 5: System dynamics showing periodic solutions orbiting around the steady state for $X(0)=0.249 ; Y(0)=0.029 ; r_{1}=0.88 ; a=0.298$; $b=0.29 ; c=0.035 ; r_{2}=0.439 ; e=0.143 ; \tau=8.21$.

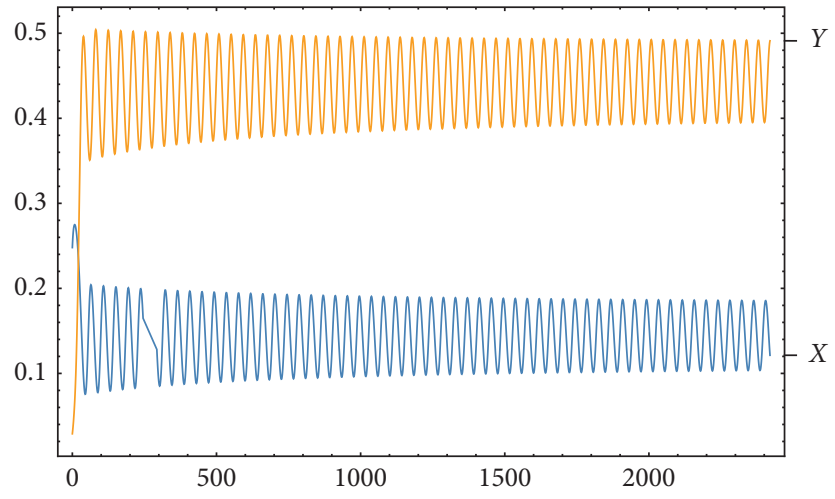

(a)

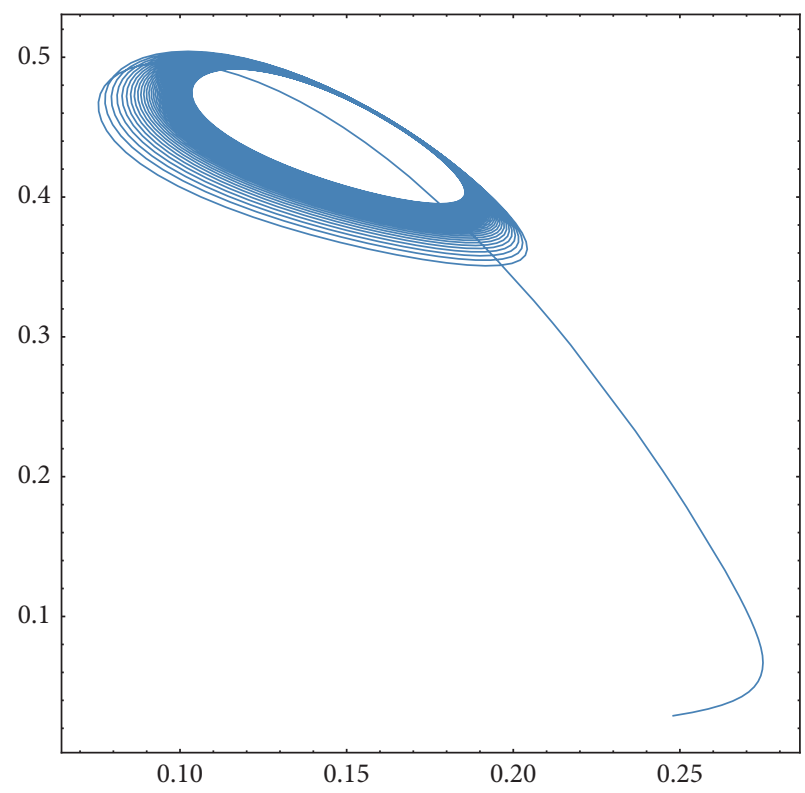

(b)

FigURE 6: System dynamics showing periodic solutions approaching a stable limit cycle for $X(0)=0.248 ; Y(0)=0.029 ; r_{1}=0.439 ; a=0.143$; $b=0.012 ; c=0.0135 ; r_{2}=0.439 ; e=0.143 ; \tau=8.21$.

relationship between the interactive entities in applied economics, electronic systems, or chemical reaction systems.

\section{Conclusion}

In this paper, we analyzed a predator-prey system with delayed interaction terms in both prey and predator equations to control the respective entity dynamics over time by promoting the predator growth over its prey for stability and security purposes. The proposed model is applied to the underlying competitive interaction occurring when a harmful rumor has to be strangled by authoritative information published for that purpose. We performed qualitative analysis of the proposed model and showed that the delay has a significant impact on the behavior of the system around its equilibrium points. The unique positive 
equilibrium point, in case of coexistence, was proven to be globally and locally asymptotically stable for some sets of parameter values and initial conditions. The system exhibited very rich dynamical behavior displaying periodic curves and a stable limit cycle at the vicinity of the steadystate equilibrium. The results of numerical simulations suggested releasing the authoritative information at the right time to maintain the stability of the system, reduce the harmful rumor spreading speed, and promote informative and yet competitive information published by the authority for social security and human wellbeing.

\section{Data Availability}

Data sharing is not applicable to this article as no new data were created or analyzed in this study.

\section{Conflicts of Interest}

The authors declare that there are no conflicts of interest regarding the publication of this paper.

\section{Acknowledgments}

This work was supported by the National Social Science Foundation of China (no. 18BXW118).

\section{References}

[1] M. Azzimonti and M. Fernandes, "Social media networks, fake news, and polarization," NBER Working Paper No. 24462, Stony Brook University, New York, NY, USA, 2018.

[2] M. Gentzkow, J. M. Shapiro, and D. F. Stone, "Media bias in the market-place: theory," in Chapter 14 in Handbook of Media Economics, S. Anderson, J. Waldofgel, and D. Stromberg, Eds., Vol. 1B, National Bureau of Economic Research, Cambridge, MA, USA, 2016.

[3] M. Jalili and M. Perc, "Information cascades in complex networks," Journal of Complex Networks, vol. 5, pp. 665-693, 2017.

[4] X. Li, Z. Wang, C. Gao, and L. Shi, "Reasoning human emotional responses from large-scale social and public media," Applied Mathematics and Computation, vol. 310, pp. 182-193, 2017.

[5] V. Capraro and M. Perc, "The evolution of trust and trustworthiness Aanjaneya Kumar," Journal of the Royal Society Interface, vol. 17, Article ID 20200491, 2020.

[6] L. Weng, J. Ratkiewicz, N. Perra, B. Goncolves, and C. Castillo, "The role of information diffusion in the evolution of social network," in KDD'13, pp. 356-364, ACM Press, New York, NY, USA, 2013.

[7] X.-P. Yan and W.-T. Li, "Hopf bifurcation and global periodic solutions in a delayed predator-prey system," Applied Mathematics and Computation, vol. 177, no. 1, pp. 427-445, 2006.

[8] Y. Song and J. Wei, "Local Hopf bifurcation and global periodic solutions in a delayed predator-prey system," Journal of Mathematical Analysis and Applications, vol. 301, no. 1, pp. 1-21, 2005.

[9] Y. Zhang, Y. H. Koura, and Y. Su, "Dynamic of a delayed predator-prey model with application to network' users' data forwarding," Scientific Reports, vol. 9, p. 12535, 2019.
[10] Na Zhang, F. Chen, Q. Su, and T. Wu, "Dynamic behaviors of a harvesting leslie-gower predator-prey model," Discrete Dynamics in Nature and Society, vol. 2011, Article ID 473949, 14 pages, 2011.

[11] X. Song and Y. Li, "Dynamic behaviors of the periodic predator-prey model with modified Leslie-Gower Hollingtype II schemes and impulsive effect," Nonlinear Analysis: Real World Applications, vol. 9, no. 1, pp. 64-79, 2008.

[12] M. A. Hixon and M. Carr, "Synergistic predation, density dependence, and population regulation in marine fish," Science, vol. 277, no. 5328, pp. 946-949, 1997.

[13] D. $\mathrm{Hu}$ and H. Cao, "Stability and bifurcation analysis in a predator-prey system with Michaelis-Menten type predator harvesting," Nonlinear Analysis: Real World Applications, vol. 33, pp. 58-82, 2017.

[14] R. P. Gupta and P. Chandra, "Bifurcation analysis of modified Leslie-Gower predator-prey model with Michaelis-Menten type prey harvesting," Journal of Mathematical Analysis and Applications, vol. 398, no. 1, pp. 278-295, 2013.

[15] C. Ji, D. Jiang, and N. Shi, "Analysis of a predator-prey model with modified Leslie-Gower and Holling-type II schemes with stochastic perturbation," Journal of Mathematical Analysis and Applications, vol. 359, no. 2, pp. 482-498, 2009.

[16] K. Vlastimil and E. Jan, "The effect of holling type II functional response on apparent competition," Theoretical Population Biology, vol. 70, pp. 421-430, 2006.

[17] S. Nakaoka, Y. Saito, Y. Saito, and Y. Takeuchi, "Stability, delay, and chaotic behavior in a Lotka-Volterra predator-prey system," Mathematical Biosciences and Engineering, vol. 3, no. 1, pp. 173-187, 2006.

[18] T. K. Kar and S. Jana, "Stability and bifurcation analysis of a stage structured predator prey model with time delay," Applied Mathematics and Computation, vol. 219, no. 8, pp. 3779-3792, 2012.

[19] D. Xiao and S. Ruan, "Global dynamics of a ratio-dependent predator-prey system," Journal of Mathematical Biology, vol. 43, no. 3, pp. 268-290, 2001.

[20] R. K. Upadhyay and S. RK. Iyengar, Introduction to Mathematical Modeling and Chaotic Dynamics, CRC Press, Boca Raton, FL, USA, First edition, 2014.

[21] S. Gakkhar and A. Singh, "Control of chaos due to additional predator in the Hastings-Powell food chain model," Journal of Mathematical Analysis and Applications, vol. 385, no. 1, pp. 423-438, 2012.

[22] B. E. Kendall, "Cycles, chaos, and noise in predator-prey dynamics," Chaos, Solitons \& Fractals, vol. 12, no. 2, pp. 321-332, 2001.

[23] T. Zhao, Y. Kuang, and H. L. Smith, "Global existence of periodic solutions in a class of delayed Gause-type predatorprey systems," Nonlinear Analysis: Theory, Methods \& Applications, vol. 28, no. 8, pp. 1373-1394, 1997.

[24] Y. Kuang, Delay Differential Equations with Applications in Population Dynamics, Academic Press, New York, NY, USA, 1993.

[25] S.-H. Ding, "Global structure of a kind of predator-prey system," Applied Mathematics and Mechanics, vol. 9, pp. 999-1003, 1988.

[26] P. Turchin, Complex Population Dynamics, Princeton University Press, Princeton, NJ, USA, 2003.

[27] D. A. Miller, J. B. Grand, T. F. Fondell, and M. Anthony, "Predator functional response and prey survival: direct and indirect interactions affecting a marked prey population," Journal of Animal Ecology, vol. 75, no. 1, pp. 101-110, 2006. 
[28] D. Xiao and Z. Zhang, "On the uniqueness and nonexistence of limit cycles for predator prey systems," Nonlinearity, vol. 16, no. 3, pp. 1185-1201, 2003.

[29] V. Krivan and J. Eisner, "The effect of holling type II functional response on apparent competition," Theoretical Population Biology, vol. 70, pp. 421-430, 2006.

[30] K. Sun, M. Shaoshuai, J. Qiu, T. Wang, and H. Gao, "Adaptive fuzzy control for nontriangular structural stochastic switched nonlinear systems with full state constraints," IEEE Transactions on Fuzzy Systems, vol. 27, no. 8, 2019.

[31] J. Qiu, T. Wang, and H. Gao, "Observer-based fuzzy adaptive event-triggered control for pure-feedback nonlinear systems with prescribed performance," IEEE Transactions on Fuzzy Systems, vol. 27, no. 11, 2019.

[32] Q. Zhou, H. Li, L. Wang, and R. Lu, "Prescribed performance observer-based adaptive fuzzy control for nonstrict-feedback stochastic nonlinear systems," IEEE Transactions on Systems, Man, and Cybernetics: Systems, vol. 48, 2018.

[33] Y. Zhang, F. Liu, Y. H. Koura et al., "Dynamic of interactive model for information propagation across social networks media," Advances in Difference Equations, vol. 2020, p. 318, 2020 . 